\title{
Quantification of Precipitation Hardening and Evolution of Precipitates
}

\author{
Zhanli Guo and Wei Sha \\ Metals Research Group, School of Civil Engineering, The Queen's University of Belfast, UK
}

Quantification of precipitation hardening is a challenging subject as it demands combined knowledge of precipitation strengthening mechanism and precipitate growth/coarsening kinetics. Having not seen many attempts on developing new theories in recent years, the authors are aware of the fact that many existing concepts and developed theories are sometimes even neglected or misused. This article therefore aims to describe overview on some aspects which have not been fully addressed and/or misused. Recent developments in this subject include an accurate determination of the equilibrium precipitate fraction and interparticle spacing. The influence from precipitation fraction on precipitate coarsening and hardening kinetics was accounted for quantitatively, which allows the hardening quantification to be carried out in a more accurate manner. In addition, difficulties in quantification of precipitation strengthening effects in commercial systems are discussed. Advanced theories on dislocation precipitate interaction mechanisms are required to improve the accuracy in quantification of precipitation hardening to a high level.

(Received October 30, 2001; Accepted April 5, 2002)

Keywords: precipitation hardening, precipitation kinetics, computer modelling, precipitate growth, precipitate coarsening

\section{Introduction}

Precipitation strengthening remains one of the most effective ways in the development of ultrahigh-strength alloys. It is achieved by producing a particulate dispersion of obstacles to dislocation movement, using a second phase precipitation process. It has been nearly a century since age hardening was first discovered by Wilm ${ }^{1)}$ (1911) in aluminium alloys. Precipitation strengthening mechanism and precipitation kinetics became the subject of research since then. ${ }^{2)}$ However, it was not until the introduction of the concept of dislocation by Mott and Nabarro ${ }^{3)}$ (1940), had the fundamental understanding of age hardening really been achieved. The equation derived several years later by Orowan ${ }^{4)}$ (1948) stands as a landmark achievement. Though having been refined since, it still serves as a basis for the theory of dispersion strengthening, or strengthening of alloys by non-deformable particles. Kelly and Nicholson ${ }^{5)}$ (1963) reviewed the early attempts at formulating theories of precipitation hardening. A review by Brown and $\mathrm{Ham}^{6)}$ (1971) discussed all the advances in understanding of the mechanisms of precipitation hardening, focusing on the ways in which dislocations interact with precipitates. Progress up to early 1980s was thoroughly discussed by Ardell, ${ }^{7)}$ who concentrated on the understanding of the statistics of dislocation-particle interactions and the mechanisms of age hardening. Though Ardell called for advances in theory, research on precipitation hardening since then has not been very extensive. Recent reviews, when mechanisms of hardening are discussed fully ${ }^{8,9)}$ or partially ${ }^{10,11)}$ still very much follow what had been discussed by Ardell. ${ }^{7}$ ) The progress in understanding of precipitation kinetics was thoroughly reviewed by Martin et al. ${ }^{12)}$ and Bratland et al. ${ }^{13)}$ For detailed information, readers are recommended to refer to Refs. 6), 7) and 12).

Quantification of precipitation hardening is a challenging subject as it demands combined knowledge of precipitation strengthening mechanism and growth/coarsening kinetics. Many attempts have been made to reproduce experimental observations through computer modelling based on the the- ories for strengthening and kinetics. ${ }^{9,14,15)}$ However, having not seen many attempts on developing new theories in recent years, ${ }^{8,10,11)}$ the authors are aware of the fact that even many existing concepts and developed theories are sometimes neglected or misused. With no attempt to review all the aspects involved in hardening mechanisms and precipitation kinetics, this article aims to clarify and highlight some aspects which have not been fully addressed, and sometimes misused. Some recent developments in this subject will be mentioned. Difficulties in quantification of precipitation strengthening effects in alloy systems are also to be discussed.

\section{Clarification of Some Concepts}

A typical one-peak precipitation-strengthening curve consists of two stages, as shown in Fig. 1. In Stage I, the resistance of a precipitate against dislocation cutting results in strength increase. In Stage II, a dislocation is forced to loop around the precipitate rather than cutting through, which also results in strength increase compared with the solution-treated material. To make the discussion easier, the term 'strengthening' is used to describe the strength increase in Stage I- the underaging period, and 'softening' for Stage II - the overaging period. It should be emphasised, though, that both mechanisms in fact lead to strength increase compared with the no-

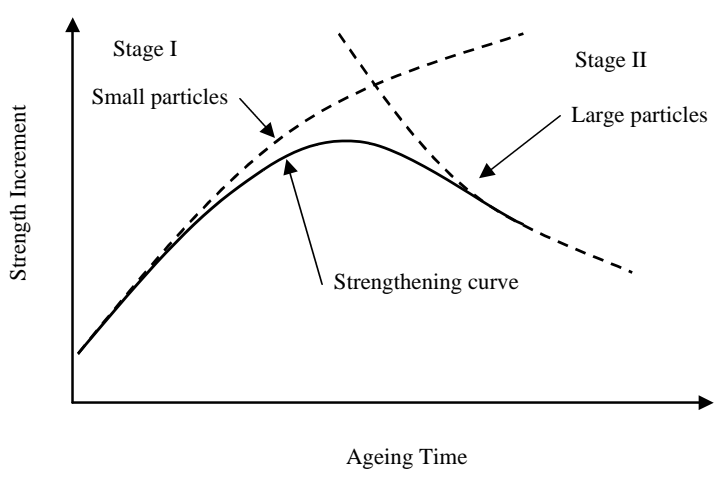

Fig. 1 A typical one-peak age hardening curve. 
particle condition and are classified as strengthening mechanisms elsewhere. The description above is not completely correct as will be explained in later sections.

\subsection{Growth and coarsening}

Precipitate growth and coarsening both need solutes from elsewhere. Solutes for growth are from the surrounding matrix, whereas for coarsening of larger precipitates they are released by the dissolving smaller particles. Such difference is clearly demonstrated in Fig. 2. However, in reality, the two sources to supply solutes that precipitates need, the surrounding matrix and the dissolving particles, usually operate simultaneously, regardless of the ongoing process being growth or coarsening. During precipitate growth, when transition phases are formed prior to the formation of a stable precipitate, the size of the stable phase increases at the expense of the transition particles. During particle coarsening, solute concentration in the surroundings of a smaller precipitate is expected to be higher than of particles of bigger size. From the view of the precipitate that grows or coarsens, the solutes are from both its surroundings and dissolved precipitates. The driving force for coarsening always consists of two parts: the difference in solute concentration and the decrease of interfacial free energy. So the diffusion processes involved in precipitate growth and coarsening have no essential difference. The difference is only in the distance of diffusion field (DoDF). The DoDF during precipitate growth decreases slightly with time if nucleation sites saturate at the beginning, and fast if more nucleation sites form during ageing. Coarsening, featured with a decrease in the number of precipitates, is always accompanied with an increase in the average DoDF. Therefore size increment during precipitate coarsening always proceeds at a rate slower than that during particle growth.

\subsection{Spinodal decomposition and normal nucleation}

There are two types of phase change in solid solutions. First there is the normal precipitation reaction which involves a thermally activated nucleation step, and secondly there is spinodal decomposition in which the material is inherently unstable to small fluctuations in composition and hence decomposes spontaneously. Nucleation is associated with metastability and requires the occurrence of a relatively large composition fluctuation to surmount the energy barrier to form a nucleus of critical size which can grow further. These intense fluctuations are relatively small in spatial extent and have composition amplitudes approaching the equilibrium composition of the precipitating phase. Spinodal decomposition refers to decomposition of an unstable solution where concentration waves of small amplitude and long wavelength are selectively amplified to develop progressively a two-phase modulated structure. During spinodal decomposition the composition of the solute-enriched regions is ini- (a)

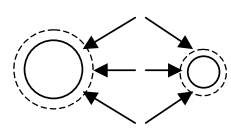

(b)

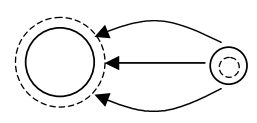

Fig. 2 Schematic comparison in diffusion routes between growth and coarsening (a) Growth, and (b) Coarsening. tially far removed from the equilibrium value.

\section{Strengthening-Critical Particle size-Coherency Strain}

\subsection{Strengthening and particle size}

Though rarely claimed, people usually have such impression that a precipitate grows during underaging, which results in hardening, and precipitate coarsens during overaging, which leads to softening. This is not correct. Precipitate growth can lead to softening, and strengthening can be achieved when a precipitate coarsens. The interaction mode between a dislocation and a particle is determined by the particle strength. The strength of a certain type of particle is related to its size. That is to say, in a sense, strengthening or softening only relates to the particle size. It is not determined by how particle reaches such size, by growth or through coarsening. If we define the critical particle size as $r_{\mathrm{c} 4}$, then:

(1) when $r<r_{\mathrm{c} 4}$, the particle is sheared by a dislocationresulting in hardening or softening;

(2) when $r>r_{\mathrm{c} 4}$, dislocations loop across the particleleading to softening. ${ }^{16)}$

The nomenclature is listed in the Appendix I.

Nicholson ${ }^{17)}$ has suggested that, irrespective of the 'hardness' of hard particles, there will be a transition to the particle cutting mechanism at very small particle sizes. Since the hardness of a small particle is rather imaginary, it is believed that understanding of such phenomenon from the view point of particle size is more convenient.

\subsection{Strengthening or softening?}

A real precipitation process is never simple. Precipitate particles can impede the motion of dislocations through a variety of interaction mechanisms, which are listed in Table 1. Among them, chemical strengthening, stacking-fault strengthening, and order hardening predict monotonic relationships between the critical resolved shear stress (CRSS) and particle radius $r$. The situation becomes complicated when coherency hardening or modulus strengthening is in control. For simplicity, we assume that the precipitation fraction has reached the equilibrium amount in the following discussion. In the case of coherency hardening, the dislocation interacts with the coherency strain field in the matrix around the coherent particle. When the particles are relatively small, one solution, relevant to very limited dislocation bending, yields that coherency strengthening increases with increasing particle size. In the case of larger particles, where considerable flexing of the dislocations occurs as a result of the spacing of individual particles, any movement of the dislocation will have to overcome a larger number of obstacles per unit length. The coherency strengthening decreases with increasing particle size. Such a relationship between strengthening effect and particle size gives rise to a maximum in the strengthening at a critical particle size $r_{\mathrm{c} 2}$ which is of the order of:

$$
r_{\mathrm{c} 2}=b /(4 \varepsilon)
$$


Table 1 Different mechanisms for precipitation hardening.

\begin{tabular}{|c|c|c|c|}
\hline Strengthening mechanism & Expression for CRSS $\Delta \tau_{\mathrm{y}}$ & Strengthening or softening & Refs. \\
\hline Chemical & $2 G\left(\frac{3}{\pi}\right)^{1 / 2}\left(\frac{\gamma_{\mathrm{s}}}{G b}\right)^{3 / 2}\left(\frac{b}{r}\right) f^{1 / 2}$ & Softening* & $\operatorname{Martin}^{11)}$ \\
\hline Stacking-fault & $\propto(\Delta \gamma)^{3 / 2} b^{-1 / 2}(r \cdot f)^{1 / 2}$ & Strengthening & $\operatorname{Martin}^{11)}$ \\
\hline \multirow[t]{2}{*}{ Modulus } & $\frac{0.9 T_{1}}{b}\left(\frac{\Delta G}{G}\right)^{\frac{3}{2}}\left(2 b \ln \left(\frac{2 r}{f^{1 / 2} b}\right)\right)^{-\frac{3}{2}}(r \cdot f)^{1 / 2}$ & Strengthening & Melander \& Persson ${ }^{18)}$ \\
\hline & Decreases when $r$ increases & Softening & Russel \& Brown ${ }^{19)}$ \\
\hline Order & $\frac{\gamma_{\mathrm{apb}}}{2 b^{2}}\left(\left(\frac{3 \pi^{2} \gamma_{\mathrm{apb}} f \cdot r}{32 T_{1}}\right)^{1 / 2}-f\right.$ & Strengthening & $\operatorname{Martin}^{11)}$ \\
\hline \multirow[t]{2}{*}{ Coherency } & $4.1 G \varepsilon^{3 / 2} f^{1 / 2}(r / b)^{1 / 2}$ & Strengthening & Gladman $^{8)}$ \\
\hline & $0.7 G \varepsilon^{1 / 4} f^{1 / 2}(b / r)^{3 / 4}$ & Softening* & Gladman $^{8)}$ \\
\hline
\end{tabular}

${ }^{*}$ These statements were made based on the assumption that precipitation fraction is nearly equilibrium. If the number of precipitates does not change in the beginning of ageing, one will have $f \propto r^{3}$ and in turn $\Delta \tau_{\mathrm{y}} \propto r^{1 / 2}$ for chemical hardening, i.e. strengthening rather than softening, and $\Delta \tau_{\mathrm{y}} \propto r^{3 / 4}$ for coherency hardening for large particles, i.e. strengthening rather than softening.

where

$$
\begin{aligned}
& \varepsilon=\left(3 K_{\theta}\left(1+v_{\alpha}\right) \delta\right) /\left(3 K_{\theta}\left(1+v_{\alpha}\right)+2 E_{\alpha}\right) \\
& \delta=2\left(\Omega_{\theta}-\Omega_{\alpha}\right) /\left(3\left(\Omega_{\theta}+\Omega_{\alpha}\right)\right)
\end{aligned}
$$

For modulus strengthening, as can be seen from Table 1, there are theories which predict a higher strength with bigger particle size. ${ }^{18)}$ However, there are also theories which predict a totally different effect, i.e. strength drop when particle grows larger. ${ }^{19)}$ There are yet no experimental data to support either theory. ${ }^{7)}$

\subsection{Evolution of particle size}

There are not just one critical particle size during the evolution of precipitate growth or coarsening. The change in the nature of coherency makes the situation even more complicated. The critical particle radius below which the particle will dissolve can be calculated as: ${ }^{20)}$

$$
r_{c}=2 c_{\alpha} \Gamma / c_{0}-c_{\alpha}
$$

where the capillarity constant $\Gamma$ is given by: ${ }^{21)}$

$$
\Gamma=\sigma N_{\mathrm{A}} \Omega_{\theta}\left(1-c_{\alpha}\right) /\left(R T\left(c_{\theta}-c_{\alpha}\right)\right)
$$

The interfacial free energy $\sigma$ will be different depending on whether the interface is coherent or incoherent, resulting in different critical radius $r_{\mathrm{c} 0}$ or $r_{\mathrm{c} 3}$. Another critical particle size $r_{\mathrm{c} 1}$ is defined to distinguish the controlling mechanism of precipitate growth in a supersaturated solid solution. The possible limiting factors considered are the rate at which atoms are brought to or removed from the interface by diffusion, and the rate at which they cross the interface. The interface reaction is likely to be the rate-controlling step during the early stages of growth since the diffusion distance tends to be zero. At large particle sizes, lattice diffusion is likely to be the slower step, since the continuous removal of solute from the matrix reduces the concentration gradient (the driving force for diffusion). A list of the critical particle sizes is given in Table 2. Since $r_{\mathrm{c} 0}$ and $r_{\mathrm{c} 1}$ are usually very small, they can be treated as zero in calculation, i.e., diffusion is assumed to be the only
Table 2 The definitions of the critical particle sizes.

$r_{\mathrm{c} 0} \quad$ Below which particle will dissolve when interface is coherent

$r_{\mathrm{c} 1} \quad$ Above which diffusion becomes the rate-controlling step

$r_{\mathrm{c} 2}$ Above which the effect of coherency strengthening decreases with increasing particle size

$r_{\mathrm{c} 3}$ Below which particle will dissolve when interface is incoherent

$r_{\mathrm{c} 4} \quad$ Above which dislocation passes particles by looping instead of shear-cutting

$r_{\mathrm{c} 5} \quad$ Above which coarsening starts to take control

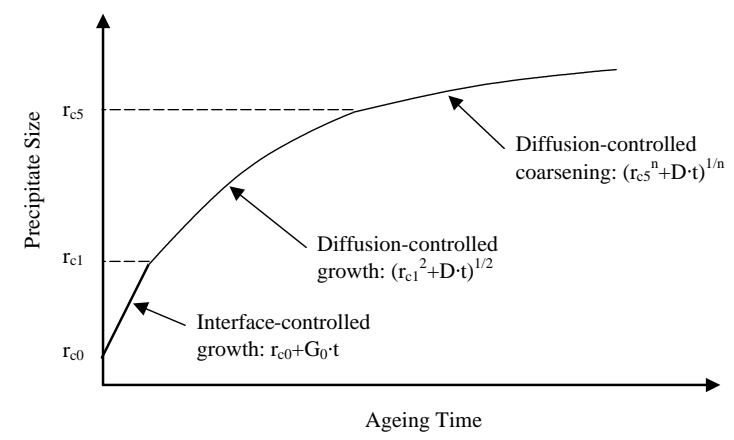

Fig. 3 Schematic diagram of the evolution of precipitate size during ageing.

controlling mechanism and the particle starts to grow at zero radius. As Kelly and Nicholson claimed that Orowan process does not occur when particles remain coherent with the matrix ${ }^{5)} r_{\mathrm{c} 3}$ should be no larger than $r_{\mathrm{c} 4}$. If the particle size at the start of coarsening is $r_{\mathrm{c} 5}$, the entire picture of the particle size evolution can be illustrated in Fig. 3. The relations between these critical sizes are $r_{\mathrm{c} 0}<r_{\mathrm{c} 1} \leq r_{\mathrm{c} 2} \leq r_{\mathrm{c} 5}$, and $r_{\mathrm{c} 0}<r_{\mathrm{c} 3}<r_{\mathrm{c} 4}$.

It is now useful to work out which is larger between $r_{\mathrm{c} 2}$ and $r_{\mathrm{c} 4}$, which will lead to a better understanding of the softening mechanism. A simple geometrical criterion for coherency given by Brooks ${ }^{22}$ is that an interface with a length $\Delta t$ remains coherent when $\Delta t \cdot \delta \leq b$, i.e. $r_{\mathrm{c} 4}=b / 2 \delta$. If assum- 
ing $K_{\theta} \approx E_{\alpha}$, and $v_{\alpha} \approx 1 / 3$, one will have $\delta \approx 1.5 \varepsilon$, and then $r_{\mathrm{c} 4} \approx b / 3 \varepsilon$. Thus, one will always have $r_{\mathrm{c} 2}$ smaller than $r_{\mathrm{c} 4}$, which implies that coherency strengthening is always followed by a coherency softening stage before it is finally taken over by Orowan looping softening mechanism. Another possibility is that dislocation looping can take place whenever the precipitate is too big/strong for dislocation to cut through, regardless of the coherency nature of the interface between the precipitate and matrix, which is in disagreement with what was claimed by Kelly and Nicholson. ${ }^{5)}$ Although some of the above theories were understood as early as 1960s, they did not receive enough attention when quantification of precipitation hardening was carried out. ${ }^{9,14)}$

\section{Quantification of Age Hardening}

\subsection{Theoretical derivation}

Floreen and Decker found that the age hardening effects in $\mathrm{Fe}-18 \mathrm{Ni}$ maraging steels over a certain temperature range could be expressed by the simple relationship: ${ }^{23,24)}$

$$
\Delta H=K t^{n_{1}}
$$

Such hardening effect is believed to be due to the particles precipitated during ageing and theoretical analysis will be carried out below. When particles are small they are sheared by moving dislocations. Their contribution to the strength of the alloy involves a convolution of the resistance to shear of one particle, their population, and the flexibility of the dislocations with which they interact, known as the 'Friedel effect'. ${ }^{25)}$ Many studies have been built on Friedel's idea, and have tested it experimentally. ${ }^{26-28)}$ For present purposes, it is adequate to accept that the contribution to the yield strength, $\Delta \sigma_{\mathrm{y}}$, of a volume fraction $f$ of shearable particles of radius $r$ has the form:

$$
\Delta \sigma_{\mathrm{y}}=c_{1} f^{m_{1}} r^{m_{2}}
$$

For most dislocation-particle interactions, both $m_{1}$ and $m_{2}$ have the value $0.5 .^{9)}$ Sensitivity analysis of the complete process model shows that $\Delta \sigma_{\mathrm{y}}$ is insensitive to the values of $m_{1}$ and $m_{2}$ in the vicinity of 0.5 . $^{9)}$ The relationship between ageing time $t$ and radius $r$ of zone or precipitate (assumed to be spherical) is given by Zener's ${ }^{29)}$ parabolic relationship:

$$
r=\alpha(D t)^{1 / m_{3}}
$$

where $m_{3}=2$. Based on different assumptions, Aaron et al. derived different equations which, though, only differ from eq. (5) in the coefficient $\alpha$ term. ${ }^{30)}$ The development of this theory and calculation of $\alpha$ under different circumstances are reviewed in Christian's book. ${ }^{21)}$

The Johnson-Mehl-Avrami (JMA) equation ${ }^{31-33)}$ can be used to describe the relationship between transformation fraction and time at a certain temperature. At early ageing stage, i.e., when $k t \ll 1$, it takes the following form:

$$
f=f_{\text {eq }}\left\{1-\exp \left[-(k t)^{m_{4}}\right]\right\} \approx f_{\text {eq }}(k t)^{m_{4}}(\text { when } k t \ll 1)
$$

For maraging steels, the increase in yield stress is proportional to the hardness increase. ${ }^{34)}$ After applying the Taylor factor $M_{\mathrm{T}}$ for polycrystalline materials, one has

$$
\Delta \sigma_{\mathrm{y}}=q \Delta H=M_{\mathrm{T}} \Delta \tau_{\mathrm{y}}
$$

Combining eqs. (4)-(7), we have:

$$
\Delta H=c_{2} t^{n_{2}}
$$

where

$$
\begin{aligned}
& c_{2}=c_{1} f_{\mathrm{eq}}^{m_{1}} \alpha^{m_{2}} D^{m_{2} / m_{3}} k^{m_{1} m_{4}} / q \\
& n_{2}=m_{1} m_{4}+m_{2} / m_{3}
\end{aligned}
$$

It seems that eq. (8) is of similar form to the experimental relationship eq. (3). However, this is not always correct. As claimed for most of dislocation-particle interactions, both $m_{1}$ and $m_{2}$ have values close to 0.5 , and $m_{3}$ equals 2 . Equation ( $8 \mathrm{~b}$ ) becomes $n_{2}=m_{4} / 2+1 / 4$. As the precipitation fraction increases with time at the early stage of ageing, $m_{4}$ always takes on positive values, under no circumstances should $n_{2}$ be less than 0.25 . However experimental observations e.g. from Floreen and Decker reported values $n_{1}$ averaged at 0.22 . A possible explanation is given in the following section.

\subsection{An explanation to $n_{2}<0.25$}

It is not difficult to find out that, in deriving eq. (8), if the value of $m_{3}$ is larger than 2 , then an $n_{2}$ value smaller than 0.25 becomes possible. This assumption basically means that the precipitate size evolution does not follow the growth law but an even slower procedure. Since the low $n$ values were first reported in $\mathrm{Fe}-18 \mathrm{Ni}$ maraging systems, what happens during ageing of such grades is first examined.

Yin and $\mathrm{Li}$ and co-workers ${ }^{35,36)}$ reported that there are three stages while ageing an $\mathrm{Fe}-18 \mathrm{Ni}$ (350-grade) maraging steel at $500^{\circ} \mathrm{C}$. The spinodal decomposition firstly appears at the early stage of ageing after solution treatment at $820^{\circ} \mathrm{C}$ for $1 \mathrm{~h} ;{ }^{37)}$ then the $\mathrm{Ni}_{3}(\mathrm{Mo}, \mathrm{Ti})$ intermetallic particles containing Fe precipitate in Ni-Mo-Ti rich regions of the modulated structure by in-situ nucleation. With increasing ageing time, $\mathrm{Ni}_{3} \mathrm{Mo}$ and $\mathrm{Ni}_{3} \mathrm{Ti}$ particles coarsen significantly and may be partly dissolved back into the matrix, which can last at least to one hour. Simultaneously the $\mathrm{Fe}_{2} \mathrm{Mo}$ particles precipitate (after ageing at $500^{\circ} \mathrm{C}$ for $7 \mathrm{~h}$ ) and reverted austenite can be found. The redistribution of atoms is fast at the initial stages of ageing. After $15 \mathrm{~min}$ intermetallic compounds start to precipitate. From Floreen and Decker's experimental observation, an $\mathrm{Fe}-18 \mathrm{Ni}$ alloy has demonstrated a significant hardness increase after ageing for $15 \mathrm{~min}$ at $482^{\circ} \mathrm{C}$ following solution treatment at $815^{\circ} \mathrm{C}$. Therefore, hardening at the early stage of ageing is due to the formation of zones enriched with $\mathrm{Ni}$, Mo and Ti by spinodal decomposition, but at later stages due to the precipitation of $\mathrm{Ni}_{3} \mathrm{Mo}$ and $\mathrm{Ni}_{3} \mathrm{Ti}$ particles.

The hardening caused by spinodal decomposition was reviewed by Ardell. ${ }^{7)}$ The theory for hardening by strong diffuse particles was claimed to be suitable to describe the hardening by spinodal decomposition. Such theory leads to a relation $\Delta \tau_{\mathrm{y}} \propto L^{2 / 3} \propto f^{-1 / 3} \cdot r^{2 / 3}$. This relationship is covered by eq. (4) but the values of $m_{1}$ and $m_{2}$ differ from those for dislocation-particle interactions. The spinodal decomposition behaviour in an $\mathrm{Fe}-28.5 \mathrm{Cr}-10.6 \mathrm{Co}$ (mass \%) alloy during ageing was chosen to exemplify the evolution of the spinodal process. The work was carried out by Brenner et al. 
using field-ion microscopy and atom probe. ${ }^{38)}$ Coarsening of the microstructure occurs from the earliest time that the two developing phases could be distinguished with the field-ion microscope. The coarsening of the network structures could be fitted to a power law with time exponent as 0.14 , which corresponds an $m_{3}$ value 7.1, a much slower size increment rate than the growth law described by eq. (5). If the hardening effect is caused by spinodal decomposition, the values of $m_{1}, m_{2}$ and $m_{3}$ will differ from the typical values given in the previous section and consequently an $n_{2}$ value smaller than 0.25 is possible.

However, there are different reports on the study of the same Fe-18Ni 350-grade, which shadowed some doubts on what happens in this alloy during early ageing. Is it spinodal decomposition or normal nucleation and growth? Tewari et $a l .{ }^{39)}$ and Mazumder et al. ${ }^{40)}$ studied the evolution of precipitates in the maraging steel of grade 350 using small angle $\mathrm{x}$ ray scattering (SAXS) and transmission electron microscopy (TEM). The sequence of phase evolution at temperatures below $450{ }^{\circ} \mathrm{C}$ involves first a rhombohedral distortion of the supersaturated martensitic $\alpha$, accompanied by the appearance of a diffuse $\omega$-like structure. In the subsequent stage, welldefined $\omega$ particles form which also contain a chemical order. The growth exponent of the $\omega$ precipitates has been found to be $1 / 5$ from the SAXS results. At higher ageing temperatures, $\mathrm{Ni}_{3}(\mathrm{Ti}, \mathrm{Mo})$ phase is the first precipitating phase to appear. SAXS results have revealed a growth exponent of $1 / 3$ consistent with the coarsening model of Lifshitz-SlyozovWagner (LSW). ${ }^{41,42)}$ Although two types of precipitates form in different temperature ranges, their growth behaviour does not follow what is described by eq. (5), but a slower procedure. As a result, even though eq. (3) may be able to be used to quantify the hardening effect, the time exponent $n_{1}$ will be temperature-dependent, which seems to be different from what was observed by Floreen and Decker. ${ }^{23,24)}$

Even when the formation of precipitate follows the nucleation procedure, the growth law described by eq. (5) may not be a good representation of the precipitate growth process. This is because one or more metastable or transition phases (not included in the phase diagram) may appear prior to or in addition to the equilibrium precipitate when supersaturated solid solution decomposes. It is believed that the formation of such phases, whose crystal structure and habit plane allow them to achieve exceptionally good lattice matching with the matrix, is more favourable than that of equilibrium phase. ${ }^{43)}$ In many systems of technological importance a transition phase completely coherent with the matrix may be formed. A simple example is the Guinier-Preston (GP) zones in the $\mathrm{Al}-\mathrm{Cu}$ system. Such zones can subsequently act as nucleating sites for other metastable precipitates. The general process that occurs, therefore, when a supersaturated solid solution is aged, is the formation of a more stable product accompanied with the dissolution of less stable phases formed in the earlier stages. This basically means that even for normal nucleation process, the size increment of particles, either transition phases formed later or stable precipitate formed in the end involves the dissolution of previously formed particles. In fact, as claimed by Wendt et al. ${ }^{44)}$ based on their work using atom probe, most of the ageing curves of aluminium alloys are controlled by coarsening kinetics. The equilibrium precipitation fraction is very small, and the formation of transition (either by interface-controlled growth or by diffusioncontrolled growth) phases takes very short period.

In conclusion, whether the phase separation proceeds through the nucleation process or through spinodal decomposition, the particle size increment may not be suitably described by the growth law of eq. (5). Instead, coarsening law may be a more reasonable option in the light of physical background. An $n_{1}$ value smaller than 0.25 becomes possible. However, quantification of age hardening does not become any easier due to the complicated situation in particle coarsening, which will be discussed in the following section.

\section{Mechanism of Particle Coarsening}

When precipitation from supersaturated solid solution is complete, further ageing leads to precipitate coarsening driven by the interfacial free energy between the precipitate and the matrix - the process known as Ostwald ripening. The coarsening kinetics depend on the rate-controlling step in the process, which has been recently reviewed in detail by Martin, et al. ${ }^{12)}$ Different controlling step results in different coarsening rate. Most of the derived relations between particle size and coarsening time follow similar expressions as below:

$$
r^{n_{3}}-r_{0}^{n_{3}}=c \cdot t
$$

where $c$ is a constant. Values of $c$ under different mechanisms ${ }^{12)}$ are summarized in Table 3.

What kind of diffusion controls the particle coarsening is difficult to determine in real systems, though LSW theory is the most widely used one. Application of this theory to interpret hardness/strength data achieves fairly satisfactory agreement with experimental results. ${ }^{45,46)}$ Some attempts were made to calculate the diffusion coefficient, which is within the constant $c$. However, the calculated diffusion values are usually much faster than the lattice diffusion coefficient extrapolated from high temperature diffusion data. For instance, based on the particle-size measurement in a Ni-11.46Si (at\%) system, ${ }^{47)}$ Ardell estimated the values of diffusion coefficient to be 10 times greater than the values extrapolated from hightemperature diffusivities. ${ }^{48)}$ Recent work on quantification of age hardening during under-ageing and overaging also reported diffusivities about two orders faster than extrapolated lattice diffusion values. ${ }^{49)}$ Though it is commonly observed that the early stages of precipitation in alloys at low ageing temperatures occur at a rate seven or eight orders of magnitude greater than that expected from the extrapolation of high temperature diffusion data due to the presence of 'quenchedin' vacancies, no such claim has been made for overaging period.

The general form of coarsening kinetics as predicted by LSW theory ${ }^{41,42)}$ appears to be well supported by thirty years of experimental evidence, in spite of various theoretical objections and alterations to the rate constant and particle size distribution. The physical basis of the LSW theory is the Gibbs-Thomson equation, which assumes that the interfacial tension and density are independent of particle size. There have been some demonstration of the variation of interfacial tension with radius of curvature. Such effects become insignificant for particles greater than $1 \mu \mathrm{m}$ radius, but may be 
Table 3 Different mechanisms for particle coarsening. ${ }^{12)}$

\begin{tabular}{lccc}
\hline Controlling mechanism & $n_{3}$ & $c$ & Remark \\
\hline Lattice diffusion & 3 & $\frac{8}{9} \frac{D \sigma}{R T} c_{\alpha} V_{\mathrm{m}}^{\theta}$ & The classic LSW theory \\
\hline Interface atom transfer & 2 & $\frac{64}{81} \frac{K \sigma}{R T} c_{\alpha} V_{\mathrm{m}}^{\theta}$ & - \\
\hline Precipitation on grain boundary & 4 & $\frac{9}{32} \frac{w_{\mathrm{gb}} D_{\mathrm{gb}} \sigma T \cdot A \cdot B}{R \mathrm{gb}} V_{\mathrm{m}}^{\theta}$ & $A=\frac{2}{3}+\frac{\sigma_{\mathrm{b}}}{2 \sigma}+\frac{1}{3}\left(\frac{\sigma_{\mathrm{b}}}{2 \sigma}\right)^{3}$ \\
& & $-1 / 2 \ln \left(1 / f_{\mathrm{b}}\right)$ & Dislocation spacing larger than interparticle spacing \\
\hline $\begin{array}{l}\text { Precipitation on low angle boundary } \\
\text { (dislocation) }\end{array}$ & 5 & - & Dislocation spacing smaller than interparticle spacing \\
\hline
\end{tabular}

significant when particles are very small. ${ }^{50)}$ In precipitation hardening alloys, strengthening precipitates usually are tens of nanometers in size. Thus, one should be aware that application of this theory may not always give very good accuracy.

\section{Recent Improvements in Quantification of Age Hard- ening}

\subsection{Matrix concentration and precipitation fraction}

The initial precipitation kinetics are adequately dealt with using Shewmon's method, ${ }^{51)}$ where the mean solute concentration $c$ in the matrix decays exponentially with time, raised to a power close to unity:

$$
c(t)=c_{\alpha}+\left(c_{0}-c_{\alpha}\right) \exp \left(-t / \tau_{1}\right)
$$

where $c_{0}$ and $c_{\alpha}$ are the initial solute concentration and equilibrium concentration at the ageing temperature, respectively, and $\tau_{1}$ is a temperature-dependent time constant. The volume fraction of precipitate $f$ is directly proportional to solute loss, tending to a final equilibrium value $f_{\text {eq }}$ when $c=c_{\alpha}$, thus:

$$
f(t) / f_{\text {eq }}=\left(c_{0}-c(t)\right) /\left(c_{0}-c_{\alpha}\right)=1-\exp \left(-t / \tau_{1}\right)
$$

Alternatively, if volume fraction evolution is described by JMA equation, the mean matrix concentration will be:

$$
c(t)=\left(c_{0}-c_{\theta} f\right) /(1-f)
$$

The latter procedure appears more rational since it allows precipitation to form first and then to calculate the mean solute concentration in the matrix. As discussed by Christian, JMA equation represents a good approximation of the real precipitation process, to an accuracy within 5\% error even when the volume fraction is high. The advantage of the latter procedure is that it allows a wide range of $m_{4}$. If eq. (11) is used, i.e. $f(t)=f_{\mathrm{eq}} \cdot t / \tau_{1}$ when $t$ is small, explanation of $n_{1}$ smaller than 0.25 will again present a problem. Another advantage of the JMA equation is, although the derivation is from geometric consideration, it does take into account the overlap of diffusion field, i.e. soft impingement, whereas, in Shewmon's treatment, the diffusion field was assumed to be infinite to allow no overlap taking place. Changes in the mean solute concentration were observed by Wendt and Haasen using atom probe. ${ }^{52)}$ It was found that $\Delta c=\left(k^{\prime} t\right)^{-1 / 3}$ represented his observations very well. In fact, their experimental data can be also well described by eq. (12), which becomes $c(t) \approx c_{0}-c_{\theta} f_{\mathrm{eq}}(k \cdot t)^{m_{4}}$ when $k t$ is small. The advantage

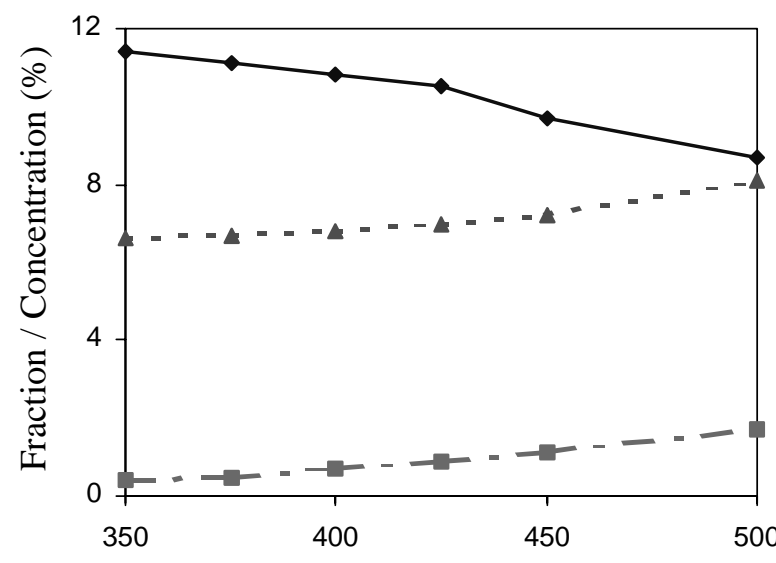

Ageing Temperature $\left({ }^{\circ} \mathrm{C}\right)$

$\longrightarrow$ Equilibrium fraction $(\mathrm{mol} \%)$

- $-\mathrm{Mn}$ in matrix (at $\%)$

- - $\Delta-=\mathrm{Ni}$ in matrix (at\%)

Fig. 4 The equilibrium fraction of the NiMn as a function of ageing temperature in an $\mathrm{Fe}-12 \mathrm{Ni}-6 \mathrm{Mn}$ system calculated from Thermo-Calc.

of eq. (12) is that it can give close approximation when $t$ approaches zero. JMA equation has been widely used to describe precipitation reactions, as well as the austenite reversion process ${ }^{53)}$ during ageing. Robino et al. ${ }^{14)}$ claimed that JMA equation may not be suitable to quantify the precipitation fraction during ageing process. However, in their quantification procedures they assumed that the number of nuclei (or precipitates) remains constant during ageing. Since this fundamental assumption cannot be justified, their conclusion that JMA equation may not be suitable for precipitation is therefore questionable.

For interstitial alloy systems containing a substitutional solute, e.g. when the precipitate type is carbide, the interface compositions $c_{\alpha}$ and $c_{\theta}$ can be calculated using the regular solution model developed by Hillert and Staffanson. ${ }^{54)}$ When the alloy system is complicated, such as commercial alloys, such thermodynamic calculations can be carried out using packages such as Thermo-Calc ${ }^{55,56)}$ and ALSTRUC. ${ }^{13)}$ The equilibrium amount of the precipitation $f_{\text {eq }}$ can be obtained through such calculations as well. The equilibrium fraction of the NiMn precipitate in an $\mathrm{Fe}-12 \mathrm{Ni}-6 \mathrm{Mn}$ system as a function of temperature is shown in Fig. 4. 


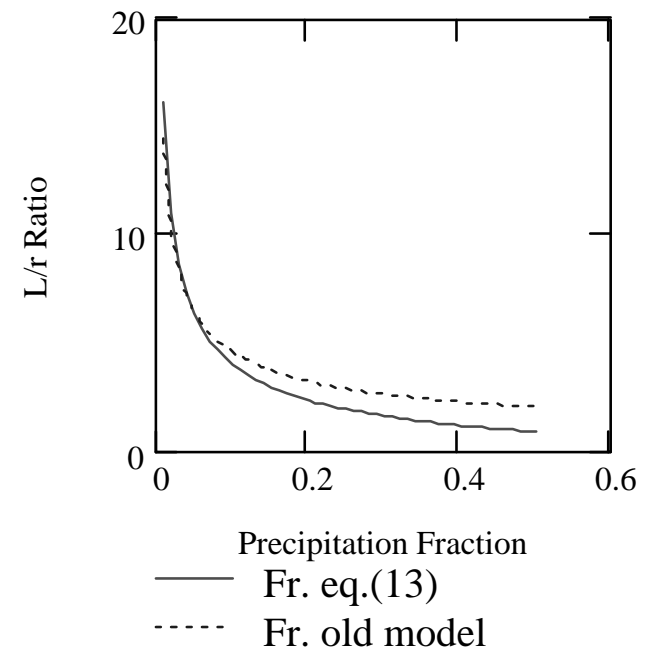

Fig. $5 \mathrm{~L} / \mathrm{r}$ ratio as a function of precipitation fraction.

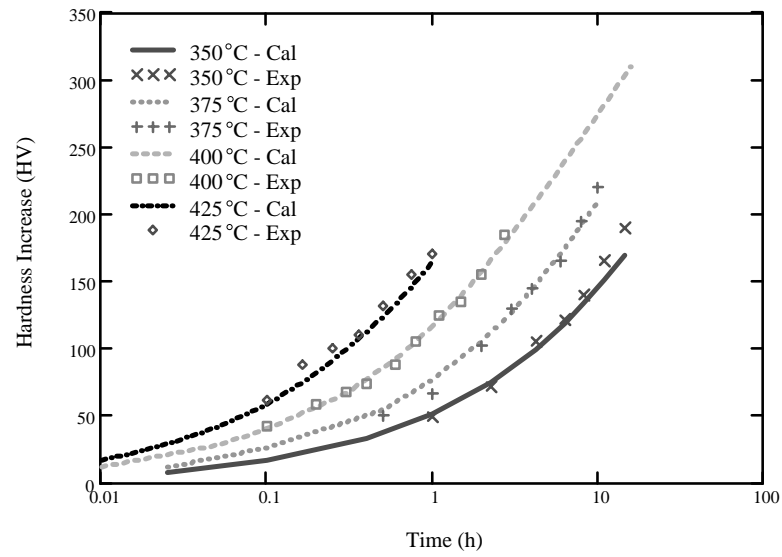

Fig. 6 Age hardening calculation and comparison at 350, 375, 400 and $425^{\circ} \mathrm{C}$.

\subsection{Calculation of interparticle spacing}

When precipitation fraction increases, the particle size should not be ignored when calculating the interparticle spacing $L$. Assuming that the precipitates are spherical, the relationship between precipitate fraction $f$, interparticle spacing and particle radius $r$ is given by; ${ }^{10,11)}$

$$
L=(1.23 \sqrt{2 \pi /(3 f)}-2 \sqrt{2 / 3}) r
$$

When precipitation fraction increases the $L / r$ ratio decreases dramatically. A comparison between eq. (13) and the old method $L / r=\sqrt{2 \pi / 3 f}$ is shown in Fig. 5. As can be seen $L$ and $r$ become very much comparable when $f$ increases to about 0.1 . The old method significantly overestimates the interparticle spacing when the precipitation fraction is large. If the volume fraction can be estimated using JMA equation, then particle spacing as a function of time will be known. Such a procedure allows the quantification of age hardening to be carried out in a more accurate manner. In the authors' previous work with an $\mathrm{Fe}-12 \mathrm{Ni}-6 \mathrm{Mn}$ alloy, the calculated hardening effects agree well with the experimental work, as shown in Fig. 6. ${ }^{49)}$

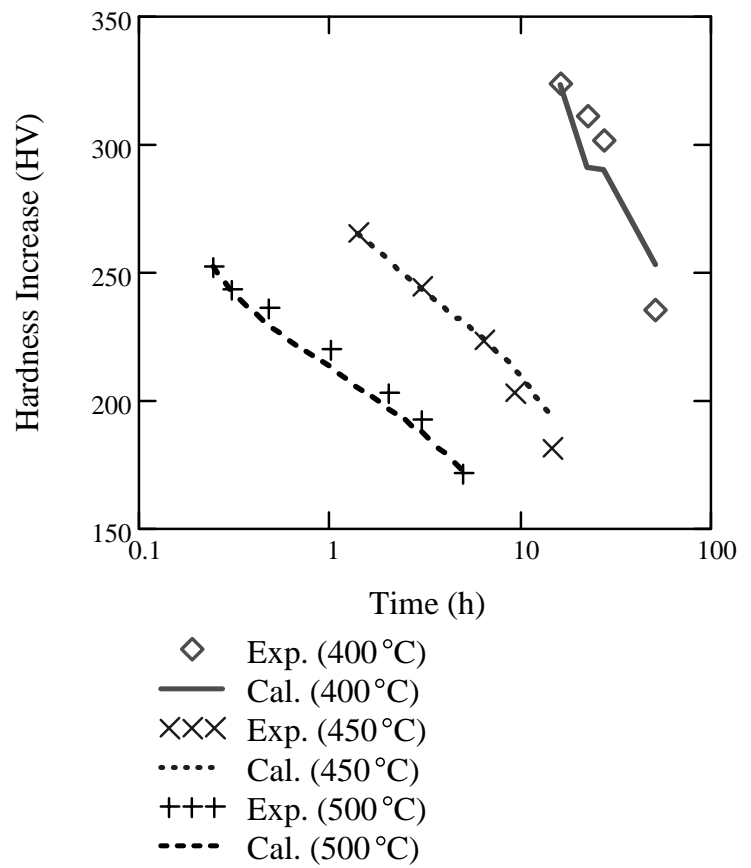

Fig. 7 Comparison between calculated and experimental results of hardening.

\subsection{Influence of volume fraction on coarsening}

Precipitation hardening consists of a few stages as shown in either Tables 4(a) or (b). Which procedure operates depends on how fast precipitation reaches the equilibrium fraction. If precipitate fraction reaches or is close to equilibrium at peak hardness, precipitation procedures follow what is listed in Table 4(a). However, in many cases the precipitation fraction does not reach equilibrium amount at peak hardness. For instance, when peak hardness is reached in an $\mathrm{Fe}-12 \mathrm{Ni}-$ $6 \mathrm{Mn}$ maraging system, only about $50 \%$ of the equilibrium amount of precipitation forms. Such precipitation behaviour follows the stages listed in Table 4(b). When quantification of age hardening is carried out, the influence from volume fraction has to be taken into account, since strictly, the relationship given by LSW theory becomes invalid when precipitation fraction deviates from zero. The effect from volume fraction $f$ on coarsening can be taken into account by introducing a parameter $k(f)$, a function of $f$. The revised LSW relationship becomes ${ }^{45}$ )

$$
r^{3}-r_{0}^{3}=M D\left(t-t_{0}\right) / R T
$$

where

$$
M=8 / 9 \cdot \sigma V_{\mathrm{m}}^{\theta} c_{\alpha}\left(1-c_{\alpha}\right) k(f) /\left(c_{\theta}-c_{\alpha}\right)^{2}
$$

When $f$ is between 0 and 0.1 , such as in the $\mathrm{Fe}-12 \mathrm{Ni}-6 \mathrm{Mn}$ system, $k(f)$ can be approximated as $k(f)=1.0+5 f .{ }^{57)}$ Such procedures have been taken into account in the authors' recent work on quantification the hardening effect during overageing of an $\mathrm{Fe}-12 \mathrm{Ni}-6 \mathrm{Mn}$ alloy. ${ }^{45)}$ Good agreement between experimental work and the calculated results was achieved, Fig. 7. 
Table 4 Stages of precipitation hardening ( $\uparrow$ for increase, and $\downarrow$ for decrease).

(a) when the equilibrium fraction is reached at peak hardness.

\begin{tabular}{ccccl}
\hline Stage & $r$ & $f$ & Number & \multicolumn{1}{c}{ Effect } \\
\hline I & $\uparrow$ & $\uparrow$ & $\uparrow$ & Strengthening \\
II & $\uparrow$ & $=f_{\text {eq }}$ & $\downarrow$ & Underaging \\
III & $\uparrow$ & $=f_{\text {eq }}$ & $\downarrow$ & Strengthening then softening \\
\hline
\end{tabular}

(b) when the equilibrium fraction is reached after peak hardness.

\begin{tabular}{ccccl}
\hline Stage & $r$ & $f$ & Number & Effect \\
\hline I & $\uparrow$ & $\uparrow$ & $\uparrow$ & Strengthening \\
II & $\uparrow$ & $\uparrow$ & $\downarrow$ & Strengthening \\
III & $\uparrow$ & $\uparrow$ & $\downarrow$ & Strengthening then softening ageing \\
IV & $\uparrow$ & $=f_{\mathrm{eq}}$ & $\downarrow$ & Softening \\
\hline
\end{tabular}

\section{Difficulties in Quantification of Age Hardening}

Quantification of precipitation hardening remains very challenging despite of a few decades of intensive research on this subject. Precipitate type determination and size and fraction measurement become possible with the aid of advanced characterization techniques such as atom probe field-ion microscopy (APFIM) ${ }^{58)}$ However, many difficulties, practical and theoretical, still exist, some of which are discussed below.

\subsection{Difficulties in the determination of the diffusion co- efficient}

There is yet a direct method to measure the diffusion coefficient at low temperatures. As a result, quantification of age hardening always features with an optimisation procedure, i.e. some parameters are determined through fitting calculated results with experimental observations. The diffusivity value obtained through this way is usually much faster than what is extrapolated from high temperature data. Although it has been recognized that diffusion during the very early stage of ageing can be very fast due to the high concentration of vacancies caused by water quenching, the fast diffusion during overaging can not be accounted for by this explanation. Without a reliable way of measuring diffusion coefficient, attempts to quantify precipitation hardening will always be semi-empirical. Another difficulty caused by the 'abnormal' diffusion is that the age hardening during underaging period can not be quantified with a constant diffusion coefficient (at a certain temperature). An experimental manifestation of such effect is that the measured as-quenched hardness differs noticeably from what is extrapolated from the hardness points during underaging period. Such phenomenon has been observed in many alloy systems, such as PH13-8Mo, ${ }^{13)} \mathrm{Fe}-$ $18 \mathrm{Ni}$ Grade-350, ${ }^{59)}$ and PH15-5 ${ }^{60)}$ steels. As a result quantification of age hardening, without taking into account the varying diffusion coefficient, encounters difficulty unsurprisingly. ${ }^{14)}$

\subsection{What is the controlling mechanism?}

Though many mechanisms were proposed theoretically to describe the particle coarsening process, no matching experimental evidence has been obtained, except for the LSW theory which found very good agreement with experimental observations in Ni-base alloys with $\gamma^{\prime}$ precipitates. ${ }^{61)}$ It is also noted that when eq. (9) is used to fit experimental results, the fitting accuracy does not change significantly when $n_{3}$ varies from 2 to 5 or even higher. ${ }^{62,63)}$ Solely from the accuracy of fitting to determine the diffusion mechanism is not convincing $^{61)}$ considering that each data point usually has some experimental error. Determination of the controlling mechanism of precipitation kinetics remains difficult experimentally and so does theoretical prediction.

Dislocation looping is the most widely used mechanism to interpret the softening effect during overaging. In fact, as discussed before, besides dislocation looping, large coherent particles and modulus difference between particle and matrix can also lead to softening. There is mounting evidence that modulus hardening may be an important mechanism of overaging in several alloy systems. Overaging is definitely not the result of the Orowan mechanism in the overaged alloys studied by Russell and Brown, ${ }^{19)}$ and Ibrahim and Ardell. ${ }^{64)}$ An evidence for Orowan looping and cross slip is a considerable enhancement of work hardening. As experimental study showed no sign of enhancement in work hardening for PH138 Mo during overaging, ${ }^{65)}$ it is unlikely that Orowan looping is the controlling softening mechanism.

\subsection{Uncertainty in the calculation of line tension}

All the strengthening mechanisms involve the calculation of dislocation line tension $T_{1}$. Its value is usually taken as $G b^{2} / 2$, quoted from Brown's approximation for fixed line tension. ${ }^{6)}$ The accuracy of this expression, as given by Brown and $\mathrm{Ham},{ }^{6}$ ) is of order-of-magnitude. Experimental result is usually lower than this value. ${ }^{7)}$ Since almost all the quantification of age hardening of a real system features with optimisation procedures, the error caused by such approximation will be transferred to other parameters such as diffusion coefficient. The calculated results based on the optimised parameters may agree very well with experimental observations, but this does not necessarily suggest a perfect model. In extreme cases, a good agreement between experimental and calculated values can be generated based on a totally wrong model. Nevertheless, such quantification procedures may be of some practical use. An accurate estimation of the line tension requires analytical theories covering the spectrum of dislocation precipitate interaction mechanisms, and computer simulations of dislocation motion through arrays of localised, but finite obstacles. Until such efforts have been made, the uncer- 
tainties in quantification of precipitate hardening will remain.

\section{Concluding Remarks}

Precipitation in metals has long been a common strengthening method. This overview has discussed some aspects which have not been fully understood or addressed in previous research and review. Theoretical analysis was given to interpret the hardening exponent. Evolution of precipitate size during ageing was addressed. The size increment of particle during early ageing period may follow a slower procedure than the classical parabolic growth law would allow, regardless of whether the phase separation follows spinodal decomposition or nucleation process. When carrying out quantification of age hardening, good agreement between calculation and experimental observation does not always prove a correct or suitably applied theory. Regarding softening during overaging, mechanisms with modulus difference or large coherent particles are possible. Though sometimes it is difficult to determine the controlling step, and in turn the operating coarsening law, LSW theory may be used for practical purpose. Some recent improvements in quantification of age hardening were also discussed, including the influence of precipitate fraction. Difficulties in quantification of precipitation hardening in commercial systems were addressed.

\section{Acknowledgements}

This work was carried out within the project of 'Computer Modelling of the Evolution of Microstructure during Processing of Maraging Steels' sponsored by the Engineering and Physical Sciences Research Council, UK, under Grant No. GR/N08971. The referee is acknowledged for his comments on the improvement on the integrity and quality of this review.

\section{REFERENCES}

1) A. Wilm: Precipitation Hardening, 1st ed., ed. by J. W. Martin (Pergamon Press, Oxford, 1968) pp. 103-111.

2) R. F. Mehl and L. K. Jetter: ASM Symposium on Age Hardening of Metals, (American Society for Metals, Metals Park, OH, 1940) p. 342.

3) N. F. Mott and F. R. N. Nabarro: Precipitation Hardening, 1st ed., ed. by J. W. Martin (Pergamon Press, Oxford, 1968) pp. 196-200.

4) E. Orowan: Precipitation Hardening, 1st ed., ed. by J. W. Martin (Pergamon Press, Oxford, 1968) pp. 201-202.

5) A. Kelly and R. B. Nicholson: Precipitation Hardening, 1st ed., ed. by J. W. Martin (Pergamon Press, Oxford, 1968) pp. 208-213.

6) L. B. Brown and R. K. Ham: Strengthening Methods in Crystals, ed. by A. Kelly and R. B. Nicholson, Chapter 2, (Elsevier, Amsterdam, 1971) pp. 9-135.

7) A. J. Ardell: Metall. Trans. A 16A (1985) 2131-2165.

8) T. Gladman: Mater. Sci. Tech. 15 (1999) 30-36.

9) H. R. Shercliff and M. F. Ashby: Acta Metall. Mater. 38 (1990) 17891802 .

10) J. W. Martin: Precipitation Hardening, 2nd ed., (ButterworthHeinemann, Oxford, 1998) pp. 58-59.

11) J. W. Martin: Precipitation Hardening, 2nd ed., (ButterworthHeinemann, Oxford, 1998) pp. 79-105.

12) J. W. Martin, R. D. Doherty and B. Cantor: Stability of Microstructure in Metallic Systems, 2nd ed., (University Press, Cambridge, 1997)

13) D. H. Bratland, O. Grong, H. Shercliff, O. R. Myhr and S. Tjotta: Acta Mater. 45 (1997) 1-22.

14) C. V. Robino, P. W. Hochanadel, G. R. Edwards and M. J. Cieslak: Metall. Mater. Trans. A 25A (1994) 697-704.
15) A. Deschamps and Y. Brechet: Acta Mater. 47 (1999) 293-305.

16) From theoretical point of view, this statement might be arguable. Derivation of the original Orowan equation $\Delta \tau_{\mathrm{y}}=2 G \cdot b / L^{4)}$ assumes precipitation fraction to be constant. Strength drop results from the increase of $\mathrm{L}$ caused by the increase of particle size with time. Theoretically it is possible that the number of particles increases with time if there is a fast increase in precipitation fraction with time.

17) R. B. Nicholson: Proceedings of Conference on Effects of Second Phase Particles on the Mechanical Properties of Steel, BSC/ISI Conference Held in Scarborough, (The Iron and Steel Institute, London, 1971) p. 1.

18) A. Melander and P. A. Persson: Acta Metall. 26 (1978) 267-278.

19) K. C. Russell and L. M. Brown, Acta Metall. 20 (1972) 969-974.

20) P. E. J. Rivera-Díaz-del-Castillo and H. K. D. H. Bhadeshia: Mater. Sci. Tech. 17 (2001) 30-32.

21) J. W. Christian: The Theory of Transformations in Metals and Alloys, 2nd ed., (Oxford, Pergamon, 1975) 179-181.

22) H. Brooks: Metal Interfaces, (ASM, Metals Park, OH, 1952) p. 20.

23) S. Floreen and R. F. Decker: in Source Book on Maraging Steels, ed. by R. F. Decker (ASM, Metals Park, OH, 1979) pp. 20-32.

24) R. F. Decker and S. Floreen: in Maraging Steels: Recent Developments and Applications, ed. by R. K. Wilson (TMS-AIME, Warrendale, PA, 1988) pp. 1-38.

25) J. Friedel: Dislocations, (Pergamon Press, London, 1964) p. 205.

26) J. W. Martin: Micromechanisms in Particle-hardened Alloys, (Cambridge Solid State Science Series, Cambridge University Press, 1980).

27) E. A. Starke Jr.: Mater. Sci. Eng. 29 (1977) 99-115.

28) J. C. Ion, K. E. Easterling and M. F. Ashby: Acta Metall. 32 (1984) 1949-1962.

29) C. Zener: J. Appl. Phys. 20 (1949) 950-953.

30) H. B. Aaron, D. Fainstein and G. R. Kolter: J. Appl. Phys. 41 (1970) 4404-4410.

31) W. A. Johnson and R. F. Mehl: Trans. Am. Inst. Min. Metall. Engrs. 135 (1939) 416-458.

32) M. Avrami: J. Chem. Phy. 9 (1941) 177-184.

33) J. Burke: The Kinetics of Phase Transformation in Metals, (Pergamon, Oxford, 1965) p. 46.

34) E. A. Wilson: Mater. Sci. Tech. 14 (1998) 277-282.

35) Z. D Yin, X. D. Li, H. B. Li and Z. H. Lai: Acta Metall. Sinica (in Chinese) 31 (1995) A7-13.

36) X. D. Li and Z. D. Yin: Mater. Letters 24 (1995) 235-238.

37) Mössbauer spectroscopy was used in the study. It was found that during the early stages of ageing the shape of the concentration is close to a pulse wave, which is characteristic of spinodal decomposition.

38) S. S. Brenner, P. P. Camus, M. K. Miller and W. A. Soffa: Acta Metall. 32 (1984) 1217-1227.

39) R. Tweari, S. Mazumder, I. S. Batra, G. K. Dey and S. Banerjee: Acta Mater. 48 (2000) 1187-1200.

40) S. Mazumder, D. Sen, I. S. Batra, R. Tweari, G. K. Dey, S. Banerjee, A. Sequeira, H. Amenitsch and S. Bernstorff: Phy. Rev. B 60 (2000) 822-830.

41) I. M. Lifshitz and V. V. Slyozov: J. Phy. Chem. Solids 19 (1961) 35-50.

42) C. Wagner: Z. Elektrochem. 65 (1961) 581-591.

43) E. Hornbogen: Aluminium 43 (1967) 115-121.

44) H. Wendt, Z. Liu and P. Haasen: Proc. 2nd Acta Scripta Int. Conf., Decomposition of Alloys: The Early Stages, ed. by P. Haasen, V. Gerold, R. Wagner and M. F. Ashby (Pergamon Press, Oxford, 1983) pp. 127132.

45) Z. Guo and W. Sha: Mater. Sci. Tech. 18 (2002) 529-533.

46) R. Taillard and A. Pineau: Mater. Sci. Eng. 56 (1982) 219-231.

47) E. Hornbogen and M. Roth: Z. Metallkunde 58 (1967) 842-855.

48) A. J. Ardell: in The Mechanism of Phase Transformations in Crystalline Solids, (Institute of Metals, London, 1969) pp. 111-116.

49) Z. Guo, W. Sha and E. A. Wilson: Mater. Sci. Tech. 18 (2002) 377-382.

50) G. W. Greenwood: in The Mechanism of Phase Transformations in Crystalline Solids, (Institute of Metals, London, 1969) pp. 103-110.

51) P. G. Shewmon: Diffusion in Solids, Chapter 1, (McGraw-Hill, New York, 1963).

52) H. Wendt and P. Haasen: Acta Metall. 31 (1983) 1649-1659.

53) D. Isobe: Denki Seiko 54 (1983) No. 4, 253-264.

54) M. Hillert and L. I. Staffanson: Acta Chem. Scand. 24 (1970) 3618 3626.

55) W. Sha: Mater. Sci. Tech. 16 (2000) 1434-1436.

56) W. Sha and G. D. W. Smith: Metall. Mater. Trans. A, 25A (1994) 25382539 . 
57) P. W. Voorhees and M. E. Glicksman: Acta Metall. 32 (1984) 20132030.

58) M. K. Miller, A. Cerezo, M. G. Hetherington and G. D. W. Smith: Atom Probe Field Ion Microscopy, (Oxford University Press, U.K., 1996).

59) U. K. Viswanathan, G. K. Dey and M. K. Asundi: Metall. Trans. A, 24A (1993) 2429-2442.

60) K. Ozbaysal and O. T. Inal: J. Mater. Sci. 29 (1994) 1471-1480.

61) M. C. Chaturvedi, D. J. Lloyd and D. W. Chung: Metal Science, (1976) 373-378.

62) M. L. Griffith, R. T. Huffman and J. W. Halloran: J. Mater. Res. 9 (1994) 1633-1643.

63) Z. Guo, unpublished research, School of Civil Engineering, Queen's University of Belfast, 2001.

64) I. A. Ibrahim and A. J. Ardell: Mater. Sci. Eng. 36 (1978) 139-143.

65) V. Seetharaman: Mater. Sci. Eng. 47 (1981) 1-11.

\section{Appendix: Nomenclature}

$A$ : constant involved in coarsening of precipitate on a grain boundary.

$b$ : Burgers vector of dislocation.

$B$ : constant involved in coarsening of precipitates on a grain boundary.

$c_{0}$ : concentration of the matrix before ageing.

$c, c_{1}, c_{2}$ : materials constants.

$c_{\mathrm{gb}}$ : solute concentration at a grain boundary in equilibrium with an infinitely large precipitate.

$c(t)$ : concentration of the matrix, changing during the ageing process.

$c_{\alpha}$ : solid solubility of the controlling elements in the matrix.

$c_{\theta}$ : concentration of the controlling elements in the new phase.

$D$ : diffusion coefficient.

$D_{\mathrm{gb}}$ : diffusion coefficients in a grain boundary.

$E_{\alpha}$ : Young's modulus of the matrix.

$f$ : volume fraction of the transformed particles.

$f_{\mathrm{b}}$ : fraction of the grain boundaries covered by precipitates.

$f_{\text {eq }}$ : equilibrium fraction of the precipitation (temperature dependent).

$f(t)$ : precipitation fraction as a function of the ageing time.

$G$ : shear modulus of the matrix.

$G_{0}$ : growth coefficient during interface-controlled growth.

$k$ : reaction rate constant in Johnson-Mehl-Avrami equation.

$k(f)$ : precipitation fraction factor in particle coarsening.

$K$ : temperature dependent rate constant.
$K_{\theta}$ : bulk modulus of the precipitate.

$L$ : interparticle spacing.

$m_{1}, m_{2}$ : exponents of $f$ and $r$ in particle shearing equation.

$m_{3}$ : reciprocal of the time exponent in the growth law.

$m_{4}$ : Avrami index in Johnson-Mehl-Avrami equation.

$M$ : parameter related to particle coarsening.

$M_{\mathrm{T}}$ : Taylor factor.

$n_{1}, n_{2}$ : time exponents in the relationship between the increase in hardness and ageing time.

$n_{3}$ : exponent in coarsening theories.

$N_{\mathrm{A}}$ : Avogadro's number.

$q$ : conversion constant between hardness and yield strength.

$R$ : gas constant.

$r$ : radius of the particle.

$r_{0}$ : particle radius at the start of coarsening.

$r_{\mathrm{c}}, r_{\mathrm{c} 0}, r_{\mathrm{c} 1}, r_{\mathrm{c} 2}, r_{\mathrm{c} 3}, r_{\mathrm{c} 4}, r_{\mathrm{c} 5}:$ critical particle sizes.

$t$ : time.

$t_{0}$ : time when a particle starts to coarsen.

$T$ : temperature (in Kelvin).

$T_{1}$ : line tension of a dislocation.

$\alpha$ : constant related to solid solubilities of the precipitate and the matrix and the concentration of the alloy.

$\gamma_{\mathrm{s}}$ : energy of a matrix-precipitate interface created by slip.

$\gamma_{\mathrm{apb}}$ : antiphase boundary energy on the slip plane of an ordered precipitate.

$\delta$ : linear strain accompanying precipitation from the matrix.

$\Delta G$ : modulus difference between the precipitate and the matrix.

$\Delta H:$ increase in hardness.

$\Delta \gamma:$ difference in stacking fault energy between the precipitate and the matrix.

$\Delta \sigma_{\mathrm{y}}$ : increase in yield strength.

$\Delta \tau_{\mathrm{y}}$ : increase in shear stress.

$\varepsilon$ : strain energy constant.

$v_{\alpha}$ : Poisson's ratio.

$\sigma$ : interface energy per unit area between the precipitate and the matrix.

$\tau_{1}$ : exponential time constant.

$\sigma_{\mathrm{b}}$ : grain boundary energy.

$\Omega_{\alpha}$ : atomic volume of the matrix.

$\Omega_{\theta}$ : atomic volume of the precipitate.

$\Gamma$ : capillarity constant. 